Jamie Toombs*a, Ross W. Paterson*a, Michael P. Lunn, Jenifer M. Nicholas, Nick C. Fox, Miles D. Chapman, Jonathan M. Schott and Henrik Zetterberg

\title{
Identification of an important potential confound in CSF AD studies: aliquot volume
}

\begin{abstract}
Background: Cerebrospinal fluid (CSF) amyloid beta 1-42 (Aß1-42), total tau (T-tau) and phosphorylated tau181 (P-tau) are finding increasing utility as biomarkers of Alzheimer's disease (AD). The purpose of this study was to determine whether measured CSF biomarker concentrations were affected by aliquot storage volume and whether addition of detergent-containing buffer mitigates any observed effects.
\end{abstract}

Methods: $\mathrm{AD}$ and control CSF was distributed into polypropylene tubes in aliquots of different volumes $(50-1500 \mu \mathrm{L}) . \mathrm{A} \beta 1-42, \mathrm{~T}$-tau and P-tau were measured with and without addition of Tween 20 (0.05\%).

Results: Measured concentrations of AB1-42 increased two-fold with aliquot storage volume. A volume increase of $10 \mu \mathrm{L}$ caused an A $\mathrm{A} 1-42$ increase of $0.95 \mathrm{pg} / \mathrm{mL}$ [95\% confidence interval (CI) 0.36-1.50, $\mathrm{p}=0.02]$ in controls, and $0.60 \mathrm{pg} / \mathrm{mL}$ (CI $0.23-0.98 \mathrm{pg} / \mathrm{mL}, \mathrm{p}=0.003$ ) in $\mathrm{AD}$ samples. Following addition of Tween 20, the positive relationship between A $\beta 1-42$ and aliquot volume disappeared. T-tau and P-tau were not significantly affected.

Conclusions: CSF aliquot storage volume has a significant impact on the measured concentration of A $31-42$. The introduction of a buffer detergent at the initial aliquoting stage may be an effective solution to this problem.

Keywords: Alzheimer's disease; amyloid $\beta$; cerebrospinal fluid; tau; volume.

alamie Toombs and Ross W. Paterson contributed equally to this work.

*Corresponding authors: Jamie Toombs, Department of Molecular Neuroscience, Institute of Neurology, University College London, London, UK, Phone: +44 20 34483553, e-mail: j.toombs@ucl.ac.uk; and Ross Paterson, Dementia Research Centre, Department of Neurodegeneration, Institute of Neurology, London, UK, Phone: +44 203448 3553, e-mail: r.paterson@ucl.ac.uk

Michael P. Lunn and Miles D. Chapman: Department of Neuroimmunology, Institute of Neurology, University College London, London, UK

Jenifer M. Nicholas: Department of Medical Statistics, London School of Hygiene and Tropical Medicine, London, UK
Nick C. Fox and Jonathan M. Schott: Department of Neurodegeneration, Institute of Neurology, Dementia Research Centre, London, UK

Henrik Zetterberg: Department of Molecular Neuroscience, Institute of Neurology, University College London, London, UK; and Department of Psychiatry and Neurochemistry, Institute of Neuroscience and Physiology, the Sahlgrenska Academy at the University of Gothenburg, Gothenburg, Sweden

\section{Introduction}

Cerebrospinal fluid (CSF) amyloid beta 1-42 (Aß1-42), total tau (T-tau) and phosphorylated tau181 (P-tau) can be used to help diagnose Alzheimer's disease (AD) pathology in individuals with cognitive impairment [1]. These biomarkers are widely measured for clinical, research and drug development purposes. However there is considerable variability in collection procedures and laboratory handling methods, leading to inconsistency in the apparent concentrations of these analytes between individuals and between different laboratories [2].

Known confounding factors in the measurement of A $31-42$ and T-tau concentrations include delays in sample analysis [3], diurnal variation [4], CSF contamination with blood or breakdown of the blood brain barrier $[5,6]$ and choice of storage tube material [7]. Of the various analytes, $A \beta 1-42$ seems to be most influenced by such external factors, due at least in part to the hydrophobic nature of $A \beta$ and its propensity to be adsorbed to the walls of collection containers, as well as to aggregate with itself and other proteins [5]. The use of polypropylene (PP) tubes mitigates the problem of adsorption to a large extent, but does not guarantee satisfactory pre-analytic behaviour as most PP tubes are made of copolymers to which $\mathrm{A} \beta$ adsorbs to different degrees depending on the tube manufacturer [7].

We hypothesised that apparent A 1 1-42, T-tau and P-tau concentrations could also be altered by varying the aliquot volume in which they are stored, and conjectured that increasing the ratio of CSF volume to surface area of PP storage container would result in decreased analyte adsorption. Furthermore, we explored whether we could mitigate any such effects by pre-treating with 
a buffer-containing detergent that would lead to reduced tube surface adsorption and more complete measurement of analyte concentrations.

\section{Materials and methods}

\section{Sample pools}

CSF aliquots of different volumes, collected and stored in PP tubes at $-80^{\circ} \mathrm{C}$ according to standardised operating procedures [1], were tested for A 1 1-42, T-tau, and P-tau. Experiments were conducted in five rounds. In each round, two pools of CSF were used: the first, from a cohort of de-identified subjects with CSF biomarker profiles consistent with $\mathrm{AD}$. These were identified according to cut-offs that, taken together, are 90\% sensitive and specific for $\mathrm{AD}[8]$ : A $\beta 1-42<530 \mathrm{ng} / \mathrm{L}$, T-tau $>350 \mathrm{ng} / \mathrm{L}$, P-tau $>60 \mathrm{ng} / \mathrm{L}$. An exception had to be made for the AD pool used in Rounds 4 and 5 due to insufficient quantities accessible. This pool was composed of CSF that met the criteria for A 1 1-42 and T-tau, but not P-tau. The second pool was composed of de-identified non-AD control (CTRL) CSF (all three biomarker concentrations within normal range). Pooled CSF was used due to the large CSF volume requirement of the study design. Different CSF pools were used over the rounds: Round 1 was unique and contained only neat CSF, Rounds 2 and 3 were identical except for the addition of a detergent buffer [Tween $20(0.05 \%)$ ] to the CSF of Round 3. Rounds 4 and 5 used identical pools, and included AD and control, neat and Tween, individual and pooled CSF types all in the same plate in order to verify comparability of results.

To confirm that pooled CSF would behave in the same way as that of individual patients, a series of aliquots of differing volumes was created from the CSF of single subjects (IND) and tested alongside the pooled samples.

\section{Sample treatment}

Pools were created from individual samples of CSF taken by lumbar puncture, which were poured into a PP $100 \mathrm{~mL}$ beaker. The CSF was mixed thoroughly for 30 min with a magnetic stirrer and then poured into a PP $50 \mathrm{~mL}$ falcon tube. This tube was centrifuged at $3000 \mathrm{~g}$ for $10 \mathrm{~min}$. CSF from the centrifuge tube was then split into two equal halves, again using $50 \mathrm{~mL}$ falcons, and $0.05 \%$ tween was added to one. Samples were aliquoted into a range of volumes $(50 \mu \mathrm{L}, 75 \mu \mathrm{L}, 100 \mu \mathrm{L}, 125 \mu \mathrm{L}, 250 \mu \mathrm{L}, 500 \mu \mathrm{L}, 1000 \mu \mathrm{L}, 1500 \mu \mathrm{L})$ and frozen at $-80^{\circ} \mathrm{C}$. This process took $1.5 \mathrm{~h}$. Every sample in this study was used on its second thaw, except the Round 2 individual subject pool which was used on its first thaw. Round 1 samples were incubated at $-80^{\circ} \mathrm{C}$ for 2 months before testing, whilst Rounds 2-5 were incubated at $-80^{\circ} \mathrm{C}$ for a week. All samples were stored in Sarstedt (Nümbrecht, Germany), $2 \mathrm{~mL}$ PP, skirted, screw top tubes (cat. 72.694.406).

Aliquots used in the A $\beta 1-42$ and T-tau plates were thawed together at room temperature for approximately $1 \mathrm{~h}$, and dispensed into disposable PP plates for dilution (1:8 and 1:2, respectively). P-tau aliquots were thawed by the same process, but not diluted. All solutions were mixed by continuous vortexing for $5 \mathrm{~s}$.

\section{Schedule and CSF analysis}

Each round of experiments was conducted separately, and run over 2 days, with AB1-42 and T-tau plates tested in parallel on the first day. P-tau was commenced on the first day, incubated overnight, and concluded on the second. A $\beta 1-42$ and T-tau were assayed on a Meso Scale Discovery 6000 platform, using MSD Human A $\beta 1-42$ and MSD Human Total Tau kits, respectively (Meso Scale Discovery, Gaithersburg, MD, USA). P-tau was run with and INNOTEST ${ }^{\circledR}$ PHOSPHO-TAU(181P) ELISA kit (Innogenetics, Ghent, Belgium), and measured using a BMG Labtech FLUOstar Omega multi-mode microplate reader. In Rounds 1, 2 and 3, samples from the AD and CTRL pools were run in triplicate on each plate, whilst those from Rounds 4 and 5 were run in duplicate due to volume restrictions. The manufacturer's protocol was followed exactly for each assay, with the exception of vortex time in the P-tau assay where $5 \mathrm{~s}$ was preferred to 10 in the interest of plate set-up speed and consistency across all assays.

\section{Statistical analysis}

Linear regression was used to examine the relationship between analyte concentration values and sample volume. The median of the measured analyte concentration values was the dependent variable and sample volume was the independent variable of interest. Pooled samples were analysed separately and, to increase power, together over multiple rounds (Supplementary data, Table 1, which accompanies the article at http://www.degruyter.com/view/j/cclm.2013.51.issue-12/issue-files/cclm.2013.51.issue-12.xml), incorporating Round as a covariate. Individual samples were used as a point of comparison, but not analysed statistically as each was only run in a single round. All analyses were conducted in Stata Version 12.1. Graphs were created using SPSS version 21.

\section{Surface area calculation}

To calculate the relative surface area (SA) per volume in the storage tube used, measurements provided by the manufacturers (Sarstedt, Nümbrecht, Germany) were used to calculate the internal surface areas at each volume. This was done by adding the lateral SA of a hollow cylinder to that of a cone. All calculations were made using the relevant calculators on www.aqua-calc.com [9].

\section{Results}

\section{$A \beta 1-42$}

For both control and AD CSF, combining Rounds 1, 2 and 4 an approximately two-fold increase in measured neat A $\beta 1-42$ concentration was observed between the largest and smallest aliquot volumes (Figure 1A). In regression analysis, for controls, an increase of $10 \mu \mathrm{L}$ aliquot volume was associated with a significant increase in $A \beta 1-42$ 

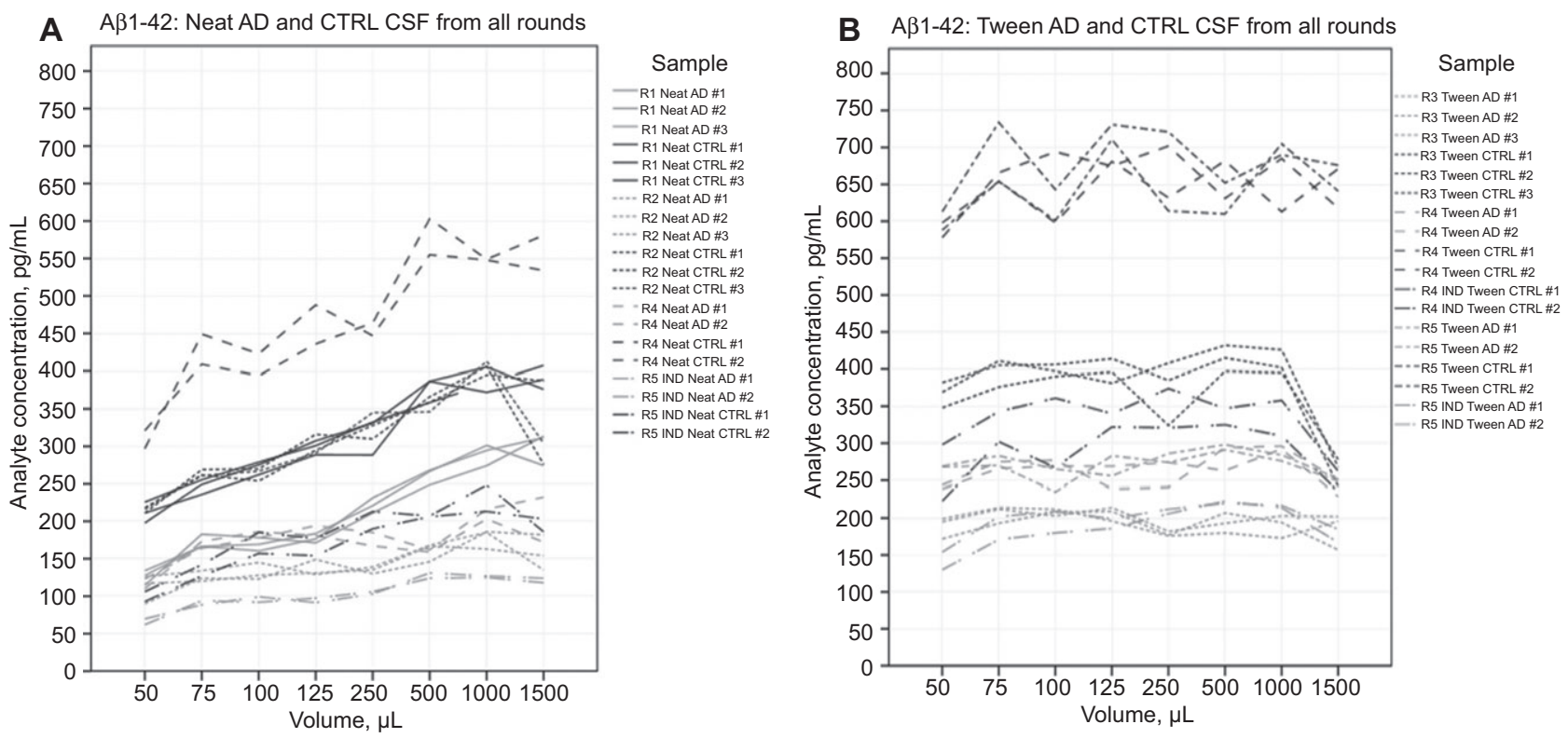

Figure 1 Shown are the results of $A \beta 1-42$ over five rounds.

(A) Results of neat pooled and individual CSF A 1 1-42, Rounds 1-5, AD and control groups. Sample R1 neat CTRL \#2 failed to react at $1000 \mu \mathrm{L}$ (signal below blank) and has been excluded. (B) Results of $0.05 \%$ Tween-treated pooled and individual CSF A $31-42$, Rounds 1-5, AD and control groups.

concentration $(0.95 \mathrm{pg} / \mathrm{mL}[95 \%$ confidence interval (CI) 0.36-1.50, $\mathrm{p}=0.02$ ]. A similar, stronger effect was seen in AD CSF: $0.60 \mathrm{pg} / \mathrm{mL}$ (CI $0.23-0.98 \mathrm{pg} / \mathrm{mL}, \mathrm{p}=0.003)$. In Rounds 3, 4 and 5 (Figure 1B), 0.05\% Tween 20 was added to all aliquots, following which there was no longer a significant relationship between aliquot volume and measured A $\beta 1-42$ concentration in either the control CSF (Supplementary data, Table 1).

\section{T-tau}

In Rounds 1, 2 and 4 (Figure 2A) there was no association between measured T-tau concentration and aliquot volume for the control pools. An increase of $10 \mu \mathrm{L}$ aliquot volume was associated with a non-significant increase in control T-tau concentration of $0.17 \mathrm{pg} / \mathrm{mL}$ (CI $-0.53-0.87 \mathrm{pg} / \mathrm{mL}, \mathrm{p}=0.06$ ). For pooled AD samples an increase of $10 \mu \mathrm{L}$ aliquot volume was associated with a non-significant increase in measured concentration of $0.05 \mathrm{pg} / \mathrm{mL}$ (CI $-1.78-1.88 \mathrm{pg} / \mathrm{mL}, \mathrm{p}=0.96$ ). For the individual samples (Figure 2D) there was no significant association between volume and measured concentration in the individuals tested. The addition of Tween 20 (Figure 2B) made no difference to the relationship between T-tau and volume.

\section{P-tau}

The results for P-tau can be seen in Figure 3. In Rounds 1, 2 and 4 (Figure $3 \mathrm{~A}$ ) there was no significant association between neat sample P-tau concentration and aliquot volume in AD CSF $(0.03 \mathrm{pg} / \mathrm{mL}$ (CI $-0.01-0.06 \mathrm{pg} / \mathrm{mL}$, $\mathrm{p}=0.10)$. In pooled control CSF samples $(0.03 \mathrm{pg} / \mathrm{mL}$ (CI $0.00-0.05 \mathrm{pg} / \mathrm{mL}, \mathrm{p}=0.03$ ), the relationship was significant when all three rounds were combined (Supplementary Data, Table 1). With the addition of Tween 20 (Figure 3B) the association between P-tau and volume disappeared.

\section{Tube surface area}

Figure 4A shows the estimated surface area of each aliquot relative to volume, and compares this to the average results of $A \beta 1-42$ at each volume. The average values were calculated from the combined A $\beta 1-42$ data of relevant (i.e., neat or Tween) individual and pooled CSF from every round of experimentation. Lower volumes are associated with higher relative surface area and lower measured concentrations of A $\beta 1-42$. This is further demonstrated by a strong inverse correlation $\left(R^{2}=0.912\right)$ between concentration of neat $A \beta 1$ 42 and relative surface area (Figure 4B). No correlation $\left(R^{2}=0.024\right)$ was found in Tween $A \beta 1-42$ samples (Figure $4 C$ ). 

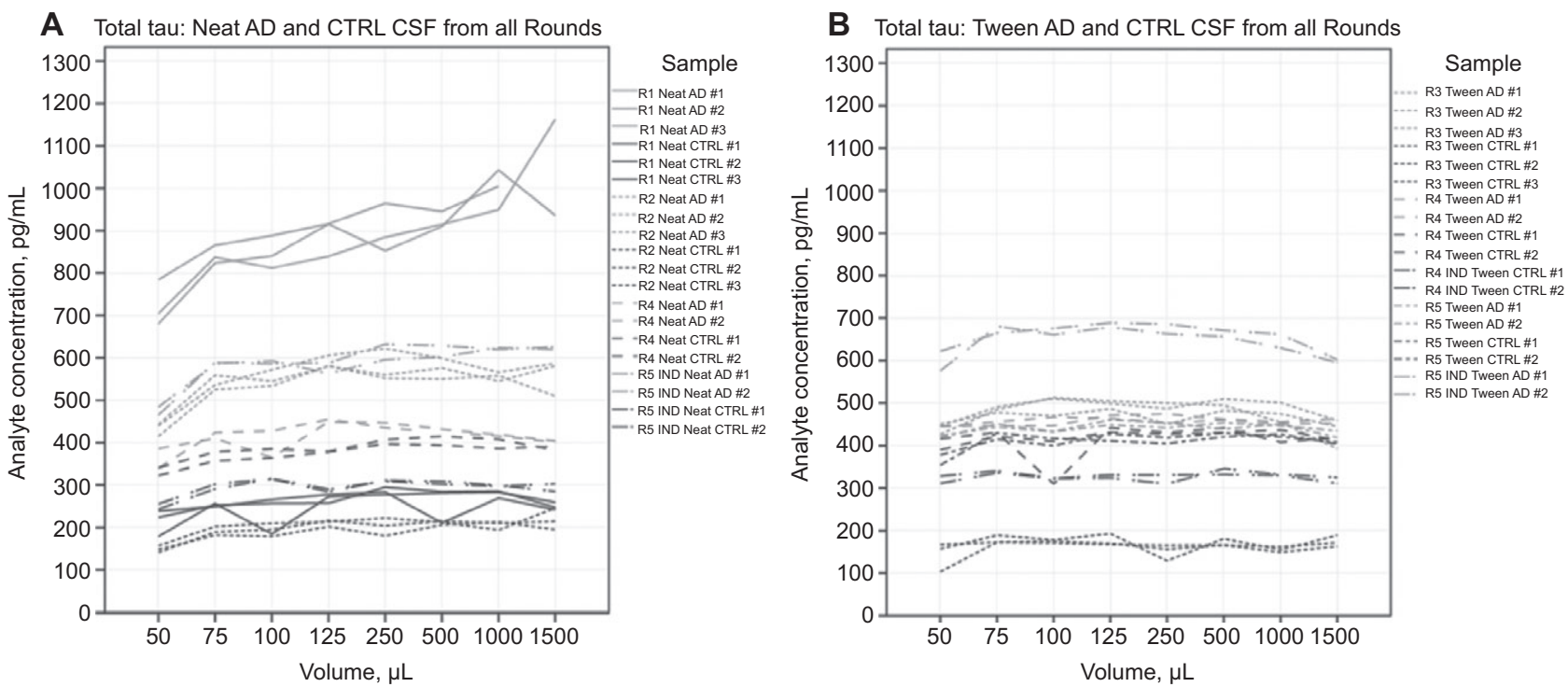

Figure 2 Shown are the results of T-tau over five rounds.

(A) Results of neat pooled and individual CSF Total tau, Rounds 1-5, AD and control groups. Samples R1 neat CTRL \#2 and R4 neat AD \#1 failed to react at $1500 \mu \mathrm{L}$ and $500 \mu \mathrm{L}$, respectively (signal below blank). These data points have been excluded. (B) Results of Tween-treated pooled and individual CSF Total tau, Rounds 1-5, AD and control groups.

\section{Discussion}

This study demonstrates that the storage volume of CSF samples has significant impact on the measured concentration of $A \beta 1-42$. The introduction of a buffer detergent to CSF samples at the initial aliquoting stage may be an effective solution to this problem. T-tau and P-tau concentrations generally appeared stable. These findings are consistent with the hypothesis that the hydrophobic properties of AB1-42 lead to adsorption of this protein to the walls of the storage vessel. In this section, we consider the impact of surface area, and other factors that may have influenced results.

\section{A Phospho-tau: Neat AD and CTRL CSF from all Rounds}

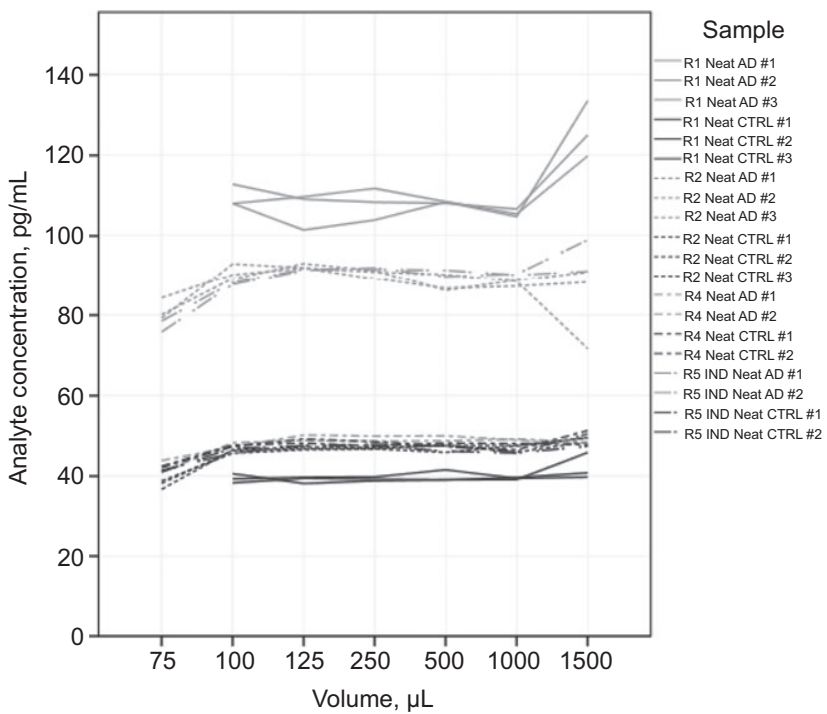

B Phospho-tau: Tween AD and CTRL from all Rounds

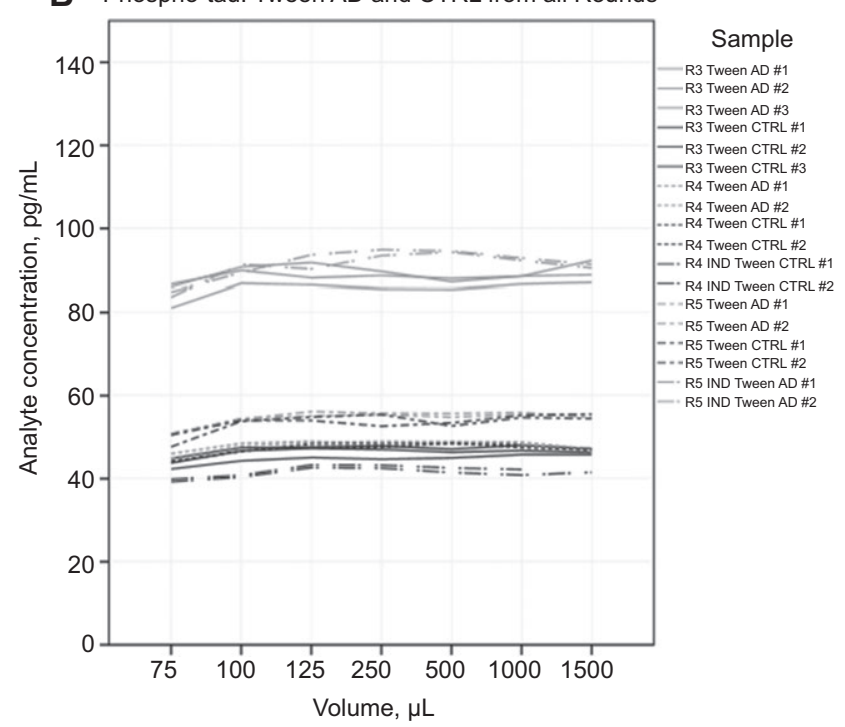

Figure 3 Shown are the results of P-tau over five rounds.

(A) Results of neat pooled and individual CSF Phospho-tau, Rounds 1-5, AD and control groups. (B): Results of Tween-treated pooled and individual CSF Phospho-tau, Rounds 1-5, AD and control groups. 

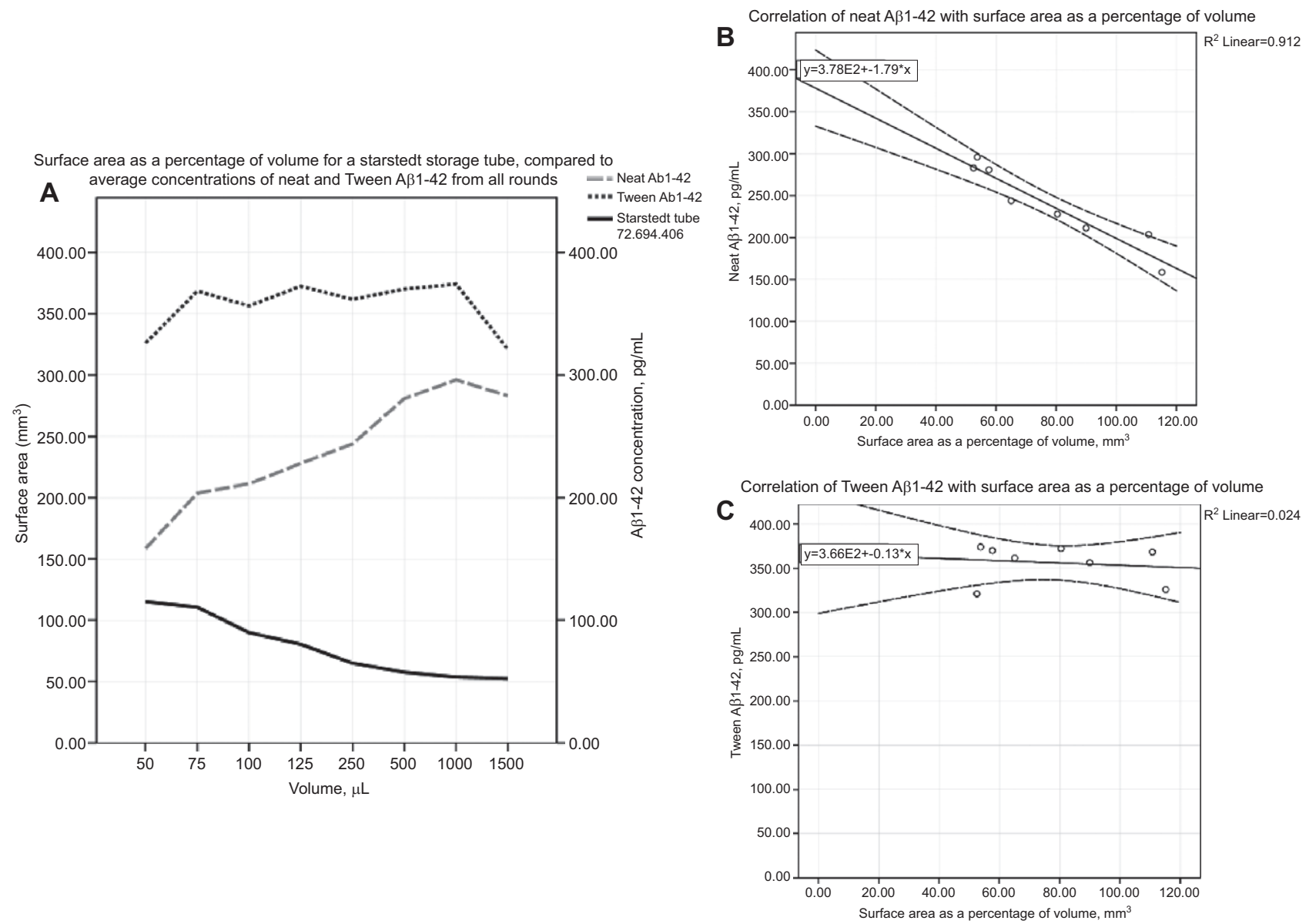

Figure 4 (A) Shown on the left y-axis are the calculated percentage values for surface area, relative to volume, for CSF in a $2 \mathrm{~mL}$ Sartstedt tube (72.694.406). Shown on the right $y$-axis are the average values, at each volume, for neat and Tween A $\beta 1-42$ combining individual and pooled CSF data from all rounds. (B) Correlation analysis of average values for neat A $31-42$ (combining individual and pooled CSF from all rounds) against surface area as a percentage of volume. Results show a strong trend for A $\beta 1-42$ concentration to decline with increased surface area. Full line=best fit. Dashed line $=95 \%$ confidence interval. (C) Correlation analysis of average values for neat $A \beta 1-42$ (combining individual and pooled CSF from all rounds) against surface area as a percentage of volume. Results show no correlation between $A \beta 1-42$ concentration and surface area. Full line=best fit. Dashed line $=95 \%$ confidence interval.

\section{Surface area}

There was an inverse relationship between the measured concentration of A $31-42$ and the surface area per volume ratio of storage tube tested (Figure 4). This suggests that surface adsorption is a key factor in the observed tendency for A $\beta 1-42$ concentration to increase with storage volume.

In the pooled AD CSF of Round 1 there was an association between volume and measured T-tau concentration, but this could not be reproduced in subsequent assays. The control and individual pools for T-tau consistently demonstrated no significant effect between volume and concentration, and this was also the case for all P-tau pools taken individually. However, when analysed in combination the neat control P-tau pool did show significant concentration increase with volume, and therefore may also be affected by surface area dependent adsorption. It must be noted that the relative increase is of much smaller magnitude than observed in A $\beta 1-42$ (expressed by the coefficient in Supplementary Data, Table 1) and is clinically irrelevant.

The frequent deviation in the concentrations of all three biomarkers which were stored in a $1500 \mu \mathrm{L}$ volume is inexplicable and disturbing. It is difficult to rationalise this as an effect of volume in regard to surface area, especially as Tween 20 samples were also affected. Instead, it may be that when volume approximates a tube's maximum capacity, the remaining space is insufficient to allow thorough mixing by the method of vortexing used. Given the magnitude of deviation across multiple samples, this tendency deserves to be explored further. 
Figure 4 shows that, although neat $A \beta 1-42$ concentration and surface area are strongly correlated, they do not perfectly correspond. Additionally, Figures 1-3 show that the magnitude of concentration increase (or stability in the case of the tau isoforms) was not always the same. Therefore it seems that whilst surface area appears to play a key role in the effect, other factors may be involved.

\section{Tween 20}

The effect of Tween 20 provides further evidence for the hypothesis that protein is lost due to surface adsorption or aggregation. Tween $20 \mathrm{~A} \beta 1-42$ samples had considerably higher concentrations of $A \beta 1-42$ than the neat samples. This suggests that a greater proportion of $A \beta 1-42$ molecules were free in the solution of the storage tube, and is consistent with the findings of other studies $[5,10,11]$. If Tween 20 were to be added routinely to samples then A $\beta 1-42$ cut points for clinical practice and clinical trials would require to be adjusted accordingly. Tween 20 tau sample results very closely reflected the results of neat samples, suggesting negligible quantities of tau are lost to surface adsorption, and that Tween 20 does not appreciably alter detection. This could suggest that the significant P-tau result may not be attributable to surface area derived adsorption.

\section{Temperature}

An alternative explanation of, or contributor to, the observed association between volume and concentration is the effect of differing thaw times. It is known that the number of freeze/thaw cycles have an effect on the protein concentration of a sample [3]. Every sample in this study was used on its second thaw or less. All aliquots were thawed together at room temperature for approximately $1 \mathrm{~h}$, but large volume samples thaw more slowly $(-80-$ approx. $21^{\circ} \mathrm{C}$ took approximately $60 \mathrm{~min}$ for a $1500 \mu \mathrm{L}$ aliquot) and so spent less time at room temperature than the low volume samples. It may be that the additional time spent at room temperature for lower volume samples allowed more proteins (particularly A $\beta 1-42$ ) to denature, become proteolysed, aggregate, or adhere. Kaiser et al. have shown that measured A $\beta 1-42$ in CSF becomes elevated over time at room temperature. In their experiment, average A $\beta 1-42$ concentration of samples frozen immediately was $790.8 \pm 329.2 \mathrm{pg} / \mathrm{nL}(\mathrm{CV}=41.6 \%)$ compared to $848.5 \pm 287.1 \mathrm{pg} / \mathrm{nL}(\mathrm{CV}=33.8 \%)$ in samples frozen after $24 \mathrm{~h}$ [12]. Tau species were not found to be significantly affected
(T-tau=304.3 $\pm 178.0 \mathrm{pg} / \mathrm{nL}$ versus $308.0 \pm 169.2 \mathrm{pg} / \mathrm{nL}$; P-tau $=59.4 \pm 29.9 \mathrm{pg} / \mathrm{nL}$ ) [13]. However, even in A $\mathrm{A} 1-42$, difference was not significant after a period of $2 \mathrm{~h}$ (only becoming so after $24 \mathrm{~h}$ ), and so unlikely to have affected our volume experiment. Additionally, the increase in concentration over time in Kaiser et al.'s study has been conjectured to be the result of cell lysis, as cells were not removed by centrifugation [5]. Bjerke et al. also reported no difference between thawing samples at room temperature, $+4^{\circ} \mathrm{C}$, and $+20^{\circ} \mathrm{C}$ in a water bath [5]. However, to our knowledge no study has examined the impact of thawing different volumes, and it remains possible that this is a factor.

\section{Further work}

Greater adsorption of $A \beta 1-42$ to vessel wall in proportion with volume to surface ratio is both credible and compatible with the results observed. The similar trend observed in the T-tau AD pool of Round 1, but not in other rounds, is not easily explained: this might suggest that T-tau is susceptible to differences in storage volume in certain circumstances (i.e., in pooling), but could also simply be a chance finding. Reflecting on the volume effect identified when P-tau rounds were combined, it is worth considering that the T-tau assay detects six isoforms of the protein, and it may be that some are more vulnerable than others to the factors previously discussed. Further studies are required to examine the extent of this phenomenon.

\section{Conclusions}

A $\beta 1-42$, T-tau and P-tau are now widely used as diagnostic markers for Alzheimer's pathology. It is, therefore, concerning that a two-fold difference can exist between the measured $A \beta 1-42$ concentrations of the $50 \mu \mathrm{L}$ and $1500 \mu \mathrm{L}$ volumes in both AD and control CSF. This could easily result in misclassification of individuals in both clinical and research settings, and is a source of variance that, to our knowledge, has not previously been considered or investigated. As we move towards 'analytical harmonisation' of CSF between centres [13], we propose that aliquot volume should also be standardised. Furthermore, as the addition of a readily available buffer detergent appears to neutralise the effect of A $\beta 1$ 42 adsorption (and potentially that of other biomarkers), the addition of Tween 20 to aliquots immediately before 
sample storage should also be explored as a practical solution to the problem.

Acknowledgements: This work was supported by the Wolfson Foundation and the NIHR Queen Square Dementia BRU. The Dementia Research Centre in an Alzheimer's Research UK Coordinating Centre. Gratitude is due to the laboratory staff at the Sahlgrenska University Hospital for providing the pooled CSF used in this study.

\section{References}

1. Blennow K, Hampel H, Weiner M, Zetterberg H. Cerebrospinal fluid and plasma biomarkers in Alzheimer disease. Nat Rev Neurol 2010;6:131-44.

2. Bartlett JW, Frost C, Mattsson N, Skillback T, Blennow K, Zetterberg H, et al. Determining cut-points for Alzheimer's disease biomarkers: statistical issues, methods and challenges. Biomark Med 2012;6:391-400.

3. Schoonenboom NS, Mulder C, Vanderstichele H, Van Elk EJ, Kok A, Van Kamp GJ, et al. Effects of processing and storage conditions on amyloid beta (1-42) and tau concentrations in cerebrospinal fluid: implications for use in clinical practice. Clin Chem 2005;51:189-95.

4. Bateman RJ, Wen G, Morris JC, Holtzman DM. Fluctuations of CSF amyloid-beta levels: implications for a diagnostic and therapeutic biomarker. Neurology 2007;68:666-9.

5. Bjerke M, Portelius E, Minthon L, Wallin A, Anckarsater H, Anckarsater $\mathrm{R}$, et al. Confounding factors influencing amyloid beta concentration in cerebrospinal fluid. Int J Alzheimers Dis 2010;2010:986310.

6. Sjogren M, Vanderstichele H, Agren H, Zachrisson O, Edsbagge M, Wikkelso C, et al. Tau and Abeta42 in cerebrospinal fluid from healthy adults 21-93 years of age: establishment of reference values. Clin Chem 2001;47:1776-81.

7. Perret-Liaudet A, Pelpel M, Tholance Y, Dumont B, Vanderstichele H, Zorzi W, et al. Cerebrospinal fluid collection tubes: a critical issue for Alzheimer disease diagnosis. Clin Chem 2012;58:787-9.

\section{Conflict of interest statement}

Authors' conflict of interest disclosure: The authors stated that there are no conflicts of interest regarding the publication of this article.

Research funding: None declared.

Employment or leadership: None declared.

Honorarium: None declared.

Received April 17, 2013; accepted July 17, 2013
8. Hansson O, Zetterberg H, Buchhave P, Londos E, Blennow K, Minthon L. Association between CSF biomarkers and incipient Alzheimer's disease in patients with mild cognitive impairment: a follow-up study. Lancet Neurol 2006;5:228-34.

9. Conversion and calculations Aqua-Calc. Available from: www. aqua-calc.com. Accessed 21 February, 2013.

10. Wiltfang J, Esselmann H, Bibl M, Smirnov A, Otto M, Paul S, et al. Highly conserved and disease-specific patterns of carboxyterminally truncated Abeta peptides 1-37/38/39 in addition to 1-40/42 in Alzheimer's disease and in patients with chronic neuroinflammation. J Neurochem 2002;81:481-96.

11. Bibl M, Esselmann H, Otto M, Lewczuk P, Cepek L, Ruther E, et al. Cerebrospinal fluid amyloid beta peptide patterns in Alzheimer's disease patients and nondemented controls depend on sample pretreatment: indication of carrier-mediated epitope masking of amyloid beta peptides. Electrophoresis 2004;25:2912-8.

12. Kaiser E, Schonknecht P, Thomann PA, Hunt A, Schroder J. Influence of delayed CSF storage on concentrations of phospho-tau protein (181), total tau protein and beta-amyloid (1-42). Neurosci Lett 2007;417:193-5.

13. Carrillo MC, Blennow K, Soares H, Lewczuk P, Mattsson N, Oberoi P, et al. Global standardization measurement of cerebral spinal fluid for Alzheimer's disease: an update from the Alzheimer's Association Global Biomarkers Consortium. Alzheimers Dement 2013;9:137-40. 